\title{
Development of nanoantibiotic delivery system using cockle shell-derived aragonite nanoparticles for treatment of osteomyelitis
}

This article was published in the following Dove Press journal:

International Journal of Nanomedicine

15 February 2016

Number of times this article has been viewed

Lamin Saidykhan'

Md Zuki Bin Abu Bakar²

Yaya Rukayadi ${ }^{1,3}$

Aminu Umar Kura ${ }^{4}$

Saiful Yazan Latifah ${ }^{5}$

'Microbiology Unit, Laboratory of Natural Products, Institute of Bioscience, ${ }^{2}$ Laboratory of Anatomy and Histology, Department of Veterinary Preclinical Sciences, Faculty of Veterinary Medicine, ${ }^{3}$ Department of Food Science, Faculty of Food Science and Technology, ${ }^{4} \mathrm{Vaccine}$ and Immunotherapeutics Laboratory Unit, Institute of Bioscience, ${ }^{5}$ Department of Biomedical Science, Faculty of Medicine and Health Sciences, Universiti Putra Malaysia, Serdang, Selangor Darul Ehsan, Malaysia
Correspondence: Md Zuki Bin Abu Bakar Laboratory of Anatomy and Histology, Department of Veterinary Preclinical Sciences, Faculty of Veterinary Medicine, Universiti Putra Malaysia, 43400 Serdang, Selangor Darul Ehsan, Malaysia

Tel +60 389473405

Fax +60 38947 I97I

Email mdzukiabubakar@gmail.com
Abstract: A local antibiotic delivery system (LADS) with biodegradable drug vehicles is recognized as the most effective therapeutic approach for the treatment of osteomyelitis. However, the design of a biodegradable LADS with high therapeutic efficacy is too costly and demanding. In this research, a low-cost, facile method was used to design vancomycin-loaded aragonite nanoparticles (VANPs) with the aim of understanding its potency in developing a nanoantibiotic bone implant for the treatment of osteomyelitis. The aragonite nanoparticles (ANPs) were synthesized from cockle shells by a hydrothermal approach using a zwitterionic surfactant. VANPs were prepared using antibiotic ratios of several nanoparticles, and the formulation (1:4) with the highest drug-loading efficiency (54.05\%) was used for physicochemical, in vitro drug release, and biological evaluation. Physiochemical characterization of VANP was performed by using transmission electron microscopy, Fourier transform infrared spectroscopy, X-ray powder diffraction, and Zetasizer. No significant differences were observed between VANP and ANP in terms of size and morphology as both samples were cubic shaped with sizes of approximately $35 \mathrm{~nm}$. The Fourier transform infrared spectroscopy of VANP indicated a weak noncovalent interaction between ANP and vancomycin, while the zeta potential values were slightly increased from $-19.4 \pm 3.3$ to $-21.2 \pm 5.7 \mathrm{mV}$ after vancomycin loading. VANP displayed 120 hours ( 5 days) release profile of vancomycin that exhibited high antibacterial effect against methicillin-resistant Staphylococcus aureus ATCC 29213. The cell proliferation assay showed $80 \%$ cell viability of human fetal osteoblast cell line 1.19 treated with the highest concentration of VANP $(250 \mu \mathrm{g} / \mathrm{mL})$, indicating good biocompatibility of VANP. In summary, VANP is a potential formulation for the development of an LADS against osteomyelitis with optimal antibacterial efficacy, good bone resorbability, and biocompatibility.

Keywords: cockle shell-derived nanoparticles, nanoantibiotics, in vitro drug release, antibacterial activity, biocompatibility

\section{Introduction}

Osteomyelitis is one of the most devastating bone diseases, especially at its chronic stage, where avascularity and loss of bone tissues are quite prevalent. ${ }^{1,2}$ Avascularity or vascular insufficiency hinders antibiotic delivery to the infected tissues, thereby rendering the administration of systemic antibiotics inefficacious. This bone infection is mainly caused by Staphylococcus aureus, and the emergence of methicillin-resistant S. aureus (MRSA) has compounded the treatment problem of osteomyelitis. The conventional treatment of osteomyelitis involves the surgical removal of destroyed bone tissues followed by intravenous supply of antimicrobials at a high concentration for 4-6 weeks. ${ }^{3}$ However, the high concentration of intravenous antibiotics can 
lead to systemic toxicity ${ }^{4,5}$ without achieving the required concentration at the affected site of infection. ${ }^{2}$

A local antibiotic delivery system (LADS) is an alternative treatment approach that has evolved to obliterate the complications associated with the conventional treatment of osteomyelitis. ${ }^{2,6}$ The mechanism of an LADS is based on the use of synthetic or natural polymers and bioceramics as antibiotic carriers to achieve the sustainable release of antibiotics at the affected site of infection. ${ }^{2,7}$ Although an LADS offers many advantages, its success is highly dependent on the properties of the materials involved in its development. Initially, poly(methyl methacrylate) cements or beads were widely used as drug delivery systems, but due to their nonbiodegradability property, they have been slowly succeeded by biomaterials that can possibly partake in bone regeneration and avoid surgical removal. ${ }^{4,5}$

A biodegradable antibiotic delivery system seemingly offers a lasting solution to the drawbacks of nonbiodegradable ones. However, since the desired drug release kinetic and bone regeneration are simultaneously difficult to achieve, ${ }^{7-9}$ designing a biodegradable delivery system becomes more demanding. The pursuit for an ideal LADS has profusely generated newer technological designs, but these designs are hampered by the high cost-to-benefit ratio. Nonetheless, the design of a biodegradable LADS using novel therapeutic agents, such as nanoantibiotics, is still regarded as a standard therapeutic option in osteomyelitis. ${ }^{8,10,11}$ Nanoantibiotics have been defined as nanomaterials that either show antimicrobial activity by themselves or elevate the effectiveness and safety of antibiotics administration. ${ }^{10}$

Cockle shells (Anadara granosa) are naturally composed of pure aragonite calcium carbonate ${ }^{12}$ and thus are a rich source of biogenic aragonite. Aragonite calcium carbonate is biocompatible and has high prospective performance in the development of advanced drug delivery system and scaffold for bone replacement and tissue engineering. ${ }^{11,13}$ In this study, a low-cost, facile method was used to design a nanoantibiotic of aragonite nanoparticles (ANPs) from cockle shells by synthesizing and incorporating the nanoparticles with vancomycin. The vancomycin-loaded aragonite nanoparticles (VANPs) were characterized by physicochemical and biological (antibacterial and biocompatibility) analyses. The aim of this study was to evaluate and understand the potency of VANP in the development of an efficient LADS for the treatment of osteomyelitis.

\section{Materials and methods}

\section{Reagents, chemicals, and media}

$N$-Dodecyl- $N, N$-dimethyl-3-ammonio-1-propanesulfonate (SB-12) was purchased from Strem Chemicals Inc.
(Newburyport, MA, USA). Mueller-Hinton agar (MHA) and Mueller-Hinton broth were obtained from Difco, Becton Dickinson (Sparks, MD, USA). Vancomycin salt, dimethyl sulfoxide, 3-(4,5-dimethylthiazol-2-yl)-2,5-diphenyltetrazolium bromide (MTT) dye, Dulbecco's modified Eagle's medium, and trypan blue dye were purchased from Sigma Chemicals (Perth, WA, Australia).

\section{Bacterial strain}

MRSA ATCC 29213 was obtained from American Type Culture Collection (ATCC; Manassas, VA, USA).

\section{Preparation of vancomycin-loaded aragonite nanoparticles Synthesis of ANPs from cockle shells}

Micron-sized powders and nanoparticles were prepared from cockle shells by employing the method of Islam et a ${ }^{14}$ with slight modifications. Briefly, the shells were cleaned by thorough scrubbing, 10 minutes boiling, drying at room temperature $\left(25^{\circ} \mathrm{C}\right)$, and rewashing with distilled water. The cleaned shells were dried in an oven at $50^{\circ} \mathrm{C}$ for 7 days. Finally, the shells were ground and sieved repeatedly using a stainless steel laboratory test sieve with an aperture size of $90 \mu \mathrm{m}$ (Endecott Ltd., London, England, UK).

Fifteen grams of micron-sized powder from cockle shells was mixed in $150 \mathrm{~mL}$ deionized water (high-performance liquid chromatography [HPLC] grade [resistance $>18 \mathrm{M} \Omega . \mathrm{cm}$ ], obtained from the Milli-RO6 plus Milli-Q-Water System [Organex]). The mixture was incubated in a water bath shaker for 24 hours at $80^{\circ} \mathrm{C}$. The ANPs were synthesized by adding $3 \mathrm{~mL}$ of SB-12 into the mixture and vigorously stirred at $1,000 \mathrm{rpm}$ at $80^{\circ} \mathrm{C}$ for 90 minutes using a systematic multi-hotplate stirrer (Witeg, WiseStir SMHS) and a magnetic stirrer bar.

The prepared ANPs were retrieved from the mixture by filtration using a double ring filter paper size of $18.0 \mathrm{~cm}$ (Filtres Fioroni, Ingré, France). The final powder fraction was dried for 2 days in an oven (Memmert UM500; GmbH \& Co., Schwabach, Germany) at $80^{\circ} \mathrm{C}$ and packed in a polyethylene plastic bag (JP Packaging, Chester, NY, USA) for subsequent experiments.

\section{Loading vancomycin on ANP}

The loading of vancomycin on ANP was performed according to the method of Chakraborty et al ${ }^{15}$ with slight modifications. Seven formulations of VANP with different nanoparticles to drug (ANP:vancomycin) ratios were prepared. Accordingly, 10, 20,30, or $40 \mathrm{mg}$ ANP was mixed in $1 \mathrm{~mL}$ phosphate-buffered saline (PBS) (pH 7.4) and sonicated for 5 minutes at $100 \mathrm{~W}$ 
with a resting interval of 15 seconds. A total of $10 \mathrm{mg}$ vancomycin was dissolved into each ANP suspension to achieve ANP:vancomycin ratios of 1:1 (VANP1), 2:1 (VANP2), 3:1 (VANP3), and 4:1 (VANP4). The ANP:vancomycin ratios of 1:2 (VANP5), 1:3 (VANP6), and 1:4 (VANP7) were prepared using the same procedure by dissolving 20,30, and $40 \mathrm{mg}$ of vancomycin into $10 \mathrm{mg}$ ANP suspension, respectively. All the suspensions were agitated at $200 \pm 1 \mathrm{rpm}$ for 48 hours at room temperature using an orbital shaker (Model 719; PROTECH, Tech-Lab Scientific Sdn. Bhd.). In total, 10-40 mg of naked ANP was prepared concurrently using a similar procedure and treated as negative control.

\section{Determination of drug-loading content and loading efficiency}

Drug-loading content and loading efficiency (LE) of VANP were determined by using the methods of Chakraborty et $\mathrm{al}^{16}$ and Zhao et $\mathrm{al}^{17}$ with slight modifications. After 48 hours of shaking, the suspensions of VANP1-7 were centrifuged at $3,500 \times g$ for 10 minutes. Supernatants from the samples were kept in a separate tube, and the pellets (VANP) were washed, dried, and kept for an in vitro drug release study. The concentrations of nonloaded vancomycin in all the supernatants were determined by using a UV/Vis spectrophotometer at a maximum wavelength of $282 \mathrm{~nm}$. The readings (absorbances) were correlated with a standard vancomycin calibration curve $\left(R^{2}=0.985\right)$, which was generated from seven vancomycin standard solutions $(40,20,10,5,2.5,1.25$, and $0.625 \mathrm{mg} / \mathrm{mL})$. The drug loading and LE were calculated using Equations 1 and 2, respectively. The analyses of drug loading and LE were expressed as the mean percentage \pm standard deviation (SD) based on two formulations that yielded similar results:

$$
\begin{aligned}
& \text { Loading content }(\%)=\frac{W_{t}-W_{f}}{W_{\mathrm{np}}} \times 100 \\
& \text { Loading efficiency }(\%)=\frac{W_{t}-W_{f}}{W_{t}} \times 100
\end{aligned}
$$

where $W_{t}$ is the total weight of drug fed, $W_{f}$ the weight of nonencapsulated free drug, and $W_{\mathrm{nP}}$ the weight of nanoparticles. ${ }^{17}$ The final results of drug loading were generated from the average of two independent experiments.

\section{Physicochemical characterization of VANP}

\section{Crystallinity and chemical properties}

The crystallinity of naked ANP, VANP, and vancomycin alone was investigated with an X-ray powder diffractometer (Shimadzu XRD-6000 powder diffractometer) using $\mathrm{CuK} \alpha$ $(\lambda=1.540562 \AA)$ at $40 \mathrm{kV}$ and $30 \mathrm{~mA} .{ }^{11}$ The crystallinity phases of the samples were determined using diffraction angles from $5^{\circ}$ to $70^{\circ}$, and the experiment was conducted at $37^{\circ} \mathrm{C}$.

Chemical analyses of naked ANP, VANP, and vancomycin alone were carried out using a Fourier transform infrared (FT-IR) spectrophotometer (Model 100 series, PerkinElmer Inc.). The spectrum was recorded at ambient temperature over a wavenumber range of $4,000-400 \mathrm{~cm}^{-1}$ at $2 \mathrm{~cm}^{-1}$ resolution ${ }^{18}$ by averaging 64 scans.

\section{Morphology and surface properties}

The shape and size distribution of ANPs and VANPs were determined by transmission electron microscopy (TEM) (Hitachi H-7100; Hitachi Ltd., Tokyo, Japan) and image analysis software, respectively. The TEM measurements were carried out at $150 \mathrm{kV}$.

The surface charges or zeta potential were analyzed by Zetasizer 4 (Malvern Instruments, Malvern, UK). Approximately $0.1 \mathrm{~g}$ of the samples were dispersed in $20 \mathrm{~mL}$ of deionized water by ultrasonication for $\sim 15$ minutes at room temperature. The prepared samples were injected into capillary cells, and zeta potential was measured by the Zetasizer at room temperature. The results were expressed as SD of three independent measurements.

\section{Drug release study and antibacterial activities}

In vitro drug release study

The profile of vancomycin released from VANP was determined using the method in PBS ( $\mathrm{pH} 7.4$ ) by adopting the method of Chakraborty et $\mathrm{l}^{15}$ with slight modifications. A total of $10 \mathrm{mg}$ of VANP was placed in a microcentrifuge tube and suspended in $2 \mathrm{~mL}$ PBS ( $\mathrm{pH}$ 7.4). The mixture was gently mixed and incubated in an incubator shaker (WiseCube ${ }^{\circledR}$ WIS-10; Wisd Laboratory Instruments, Witeg, Germany) at $37^{\circ} \mathrm{C} \pm 0.5^{\circ} \mathrm{C}$ at $120 \pm 1 \mathrm{rpm}$ for 6 days ( 144 hours). The tube was then centrifuged at 4,091 rpm for 5 minutes. At calculated time spans of 1 hour, 2-14 and 24 hours (1 day), and 48 ( 2 days) -144 hours ( 6 days), $500 \mu \mathrm{L}$ of the supernatant was withdrawn and replaced by an equal amount of fresh PBS to maintain a sink condition. The samples were aliquot, and one half was stored at $-20^{\circ} \mathrm{C}$ for the antibacterial assay. The concentration of released vancomycin was determined in real time at $282 \mathrm{~nm}$ (wavelength) using a UV/Vis spectrophotometer. The optical densities were interpreted according to a vancomycin calibration standard curve $\left(R^{2}=0.9798\right)$. The release study was conducted until there was apparently a total release of vancomycin. The results obtained from the 
three repeats were expressed as a mean $\pm \mathrm{SD}, \mathrm{n}=3$, and were represented on a graph of cumulative percentage of drug release versus time intervals.

\section{In vitro antibacterial assay}

The bactericidal kinetics of released vancomycin was analyzed according to the method of El-Ghannam et $\mathrm{al}^{7}$ with some adjustments. Vancomycin-released samples were tested against MRSA ATCC 29213 by an agar-dilution susceptibility assay according to the procedure of Clinical and Laboratory Standards Institute. ${ }^{19}$ The concentration of active or functional vancomycin was evaluated based on the antibacterial effect before and after its association with ANP (by loading). The inhibition zone was compared with a vancomycin inhibition standard curve $\left(R^{2}=0.9913\right)$ to determine their corresponding concentrations. The standard curve was plotted using ten vancomycin doses $(0.0195-20 \mathrm{mg} / \mathrm{mL})$ against their respective inhibition zone.

\section{Agar-dilution susceptibility assay}

Antibacterial activity of released vancomycin against MRSA ATCC 29213 was examined according to the methods of Ferraz et $\mathrm{al}^{9}$ and El-Ghannam et al. ${ }^{7}$ The bacterial colonies of MRSA ATCC 29213 from fresh overnight culture (on MHA) were used to prepare a $10^{6}$ colony-forming units $/ \mathrm{mL}$ suspension in Mueller-Hinton broth. The bacterial suspension was prepared according to the turbidity of $0.5 \mathrm{McF}$ arland standards. ${ }^{19}$ In total, 1 mL of MRSA ATCC 29213 suspension was incorporated into $150 \mathrm{~mL}$ of warmed $\left(45^{\circ} \mathrm{C}\right)$ nutrient agar medium (MHA) (CM3; Oxiod Ltd., Basingstoke, UK). The agar was poured in sterile petri dishes and allowed to solidify at room temperature. In each petri dish (MRSA ATCC 29213 culture plate), equidistant wells of $10 \mathrm{~mm}$ depth were created in the agar and $70 \mu \mathrm{L}$ of the released vancomycin were individually dispensed into each well. The plates were left at room temperature for 30 minutes before incubation at $37^{\circ} \mathrm{C}$. The samples obtained from the ANP suspension (correspondingly prepared in the release study) were tested against MRSA using the same susceptibility assay method and used as a negative control.

The agar diffusion tests for all samples were performed in triplicates, and the mean results were taken. After 18 hours of incubation, the inhibition zone diameter of each sample was measured and their corresponding concentration was determined according to a vancomycin inhibition standard (microbiological) curve $\left(R^{2}=0.9913\right)$. The curve was plotted on 13 standard vancomycin solutions $(0.0195-20 \mathrm{mg} / \mathrm{mL})$ with their respective inhibition zone diameters. The concentration of vancomycin before (spectrophotometric) and after (microbiological) association with ANPs was compared to evaluate the antibacterial potency of vancomycin (ie, active vancomycin). ${ }^{20}$

\section{In vitro cytotoxicity study \\ Preparation of osteoblast cell}

The human fetal osteoblast cell line 1.19 (hFOB 1.19), purchased from ATCC was used for the cytotoxicity assay. The cells were cultured in complete growth media (ie, Dulbecco's modified Eagle's medium $+10 \%$ fetal bovine serum +100 units $/ \mathrm{mL}$ penicillin and $50 \mu \mathrm{g} / \mathrm{mL}$ streptomycin) in a $5 \% \mathrm{CO}_{2}$ incubator at $37^{\circ} \mathrm{C}$. The cells were subcultured at 24 hours interval until $80 \%-90 \%$ cell confluence was achieved. After trypzination and harvesting, the cells were seeded in a $96-$ well plate and incubated at $37^{\circ} \mathrm{C}$ in the $5 \% \mathrm{CO}_{2}$ incubator for 24 hours. ${ }^{21,22}$ The authors advise that the Universiti Putra Malaysia Institutional Animal Care and Use Committee did not require ethics approval for the use of human cell lines in this study as the cell line used is a commercialized ATCC cell line.

\section{MTT assay}

The seeded osteoblasts cells were exposed to different concentrations of vancomycin, ANP, and VANP $(250,125$, $62.5,31.25$, and $15.625 \mu \mathrm{g} / \mathrm{mL}$ ) and incubated for 24,28 , and 72 hours at $37^{\circ} \mathrm{C}$ in the $5 \% \mathrm{CO}_{2}$ incubator (Thermo Fisher Scientific, Waltham, MA, USA). One well row of cell suspension was left without sample treatment and taken as a control. After the exposure was completed, the media were aspirated and replaced with maintenance media (ie, media without serum) prior to MTT treatment. Aliquots of $20 \mu \mathrm{L}$ of MTT reagent (Sigma-Aldrich Co., St Louis, MO, USA) in PBS were added into each well, and the plate was incubated at $37^{\circ} \mathrm{C}$ for 4 hours (Thermo Fisher Scientific). This allows the MTT solution to be converted to formazin by viable cells. After 4 hours of incubation, the medium was removed and replaced with $200 \mu \mathrm{L}$ of dimethyl sulfoxide. The plate was kept in a dark room for 30 minutes, and optical densities of the solutions were measured at a wavelength of $570 \mathrm{~nm}$ by the enzyme-linked immunosorbent assay reader., ${ }^{9,21}$ The percentage cell viability was calculated and expressed as percentage using the following equation:

$$
\text { Cell viability }(\%)=\frac{[\mathrm{OD}]_{\text {test }}}{[\mathrm{OD}]_{\text {control }}} \times 100 \%
$$

Here OD* is the optical density.

\section{Statistical analysis}

All experiments were performed at least three times unless otherwise indicated. The results were expressed as mean \pm SD. 
Statistical analyses were performed using SPSS software package (SPSS 14.0 for Windows; SPSS, Inc., Chicago, IL, USA). Comparisons of the means of control and experimental groups were made by one-way analysis of variance test with post hoc group comparison of Duncan's multiple range test, where $P<0.05$ was considered to be significant unless indicated otherwise.

\section{Results and discussion}

\section{Vancomycin loading and physicochemical}

\section{properties of VANP}

\section{Drug loading and LE}

The loading of vancomycin on ANP was achieved at different ANP:vancomycin ratios denoted by VANP1-VANP7. Table 1 summarizes the drug-loading content and LE of all formulations. The loading contents of VANP1, VANP2, VANP3, and VANP4 were decreased by $45.59 \%, 16.07 \%, 7.54 \%$, and $1.00 \%$, respectively, while VANP5, VANP6, and VANP7 showed increasing loading contents of $95.99 \%, 156.31 \%$, and $216 \%$, respectively. VANP7 with a ANP:vancomycin ratio of 1:4 possessed the highest drug LE $(54.05 \%)$ and was thus selected for subsequent works in our study.

From Table 1, it is apparent that LE is inversely proportional to the ANP:vancomycin ratio (the LE values reduce as ANP:vancomycin ratio increases and vice versa). The successful loading of vancomycin on ANP can be ascribed to the negative surface charge of ANP (refer to surface charge below), which promoted an electrostatic interaction between the nanoparticles and vancomycin. Being polycationic, ${ }^{23}$ vancomycin is suggested to have interacted with the negatively charged ANP through its ammonium ion $\left(\mathrm{NH}^{+}\right)$group (Figure 1) as indicated by the FT-IR results (Figure 2).

\section{X-ray powder diffraction patterns}

The X-ray powder diffraction (XRD) patterns of vancomycin, ANP, and VANP are shown in Figure 3. A hub was observed in the XRD pattern of vancomycin from $2 \theta$ of $10^{\circ}$ to $35^{\circ}$ as shown in Figure 3A. The XRD pattern for ANP showed characteristic peaks with high intensity between $2 \theta$ of $25^{\circ}$ and $55^{\circ}$. All the diffraction spectra of the nanoparticles conform with the reference spectra (JCPDS file no 00-041-1475) for aragonite polymorph of calcium carbonate. ${ }^{11}$ The high intensity of the peaks was attributed to the high crystallinity of the nanoparticles. The XRD patterns of VANP were very much similar to the naked nanoparticles; thus, we concluded that the crystallinity of the ANP was maintained after loading with vancomycin.

\section{Fourier transform infrared spectroscopy}

The FT-IR spectra of vancomycin, ANP, and VANP are presented in Figure 2. The infrared spectra of ANP (Figure 2B) showed vibrational absorption bands at $1,788 \mathrm{~cm}^{-1}$ due to $\mathrm{C}=\mathrm{O}$ stretching ${ }^{25}$ and at $1,082,860$, and $713 \mathrm{~cm}^{-1}$ due to carbonate $\left(\mathrm{CO}_{3}{ }^{2-}\right)$. The carbonate vibrational stretch is typically descriptive of the aragonite polymorph of $\mathrm{CaCO}_{3} \cdot{ }^{12,14,26}$

The FT-IR spectra of vancomycin (Figure 2A) showed vibrational bands at $3,420 \mathrm{~cm}^{-1}$, which was categorized under the phenolic $\mathrm{O}-\mathrm{H}$ group, and 1,650 $\mathrm{cm}^{-1}$, which was categorized under the aromatic $\mathrm{C}=\mathrm{C}$ double bond group. The absorption peak, 1,505 $\mathrm{cm}^{-1}$, was categorized under the amino $\left(\mathrm{NH}_{3}^{+}\right)$group as depicted in the structure of vancomycin (Figure 1).

The infrared spectra of VANP showed the presence of all absorption bands of the naked ANP without relevant shifting or stretching. No vibrational absorption bands of vancomycin were visible in VANP except at $599 \mathrm{~cm}^{-1}$, which was shifted to $562 \mathrm{~cm}^{-1}$. The FT-IR analyses of VANP implied that the interaction between ANP and VANP is weak and noncovalent. ${ }^{27}$

\section{Particle size and shape}

The morphology and particle size distribution of ANP and VANP are displayed by the TEM images (Figure 4) and the measurement of image analysis software (Figure 5), respectively. The TEM image of ANP revealed aggregated cubic-shaped nanoparticles (Figure 4A), which were visible

Table I Loading content and loading efficiency of VANP formulations

\begin{tabular}{llllll}
\hline Samples & Weight of ANP $(\mathbf{m g})$ & Weight of vancomycin $(\mathbf{m g})$ & ANP:vancomyin ratio & Loading content (\%) & Loading efficiency $(\%)$ \\
\hline VANPI & 10 & 10 & $1: 1$ & 45.59 & 45.59 \\
VANP2 & 20 & 10 & $2: 1$ & 16.07 & 32.14 \\
VANP3 & 30 & 10 & $3: 1$ & 7.54 & 22.62 \\
VANP4 & 40 & 10 & $4: 1$ & 1.00 & 4.00 \\
VANP5 & 10 & 20 & $1: 2$ & 95.99 & 50.00 \\
VANP6 & 10 & 30 & $1: 3$ & 156.31 & 52.10 \\
VANP7 & 10 & 40 & $1: 4$ & 216.18 & 54.05 \\
\hline
\end{tabular}

Abbreviations: VANP, vancomycin-loaded aragonite nanoparticle; ANP, aragonite nanoparticle. 


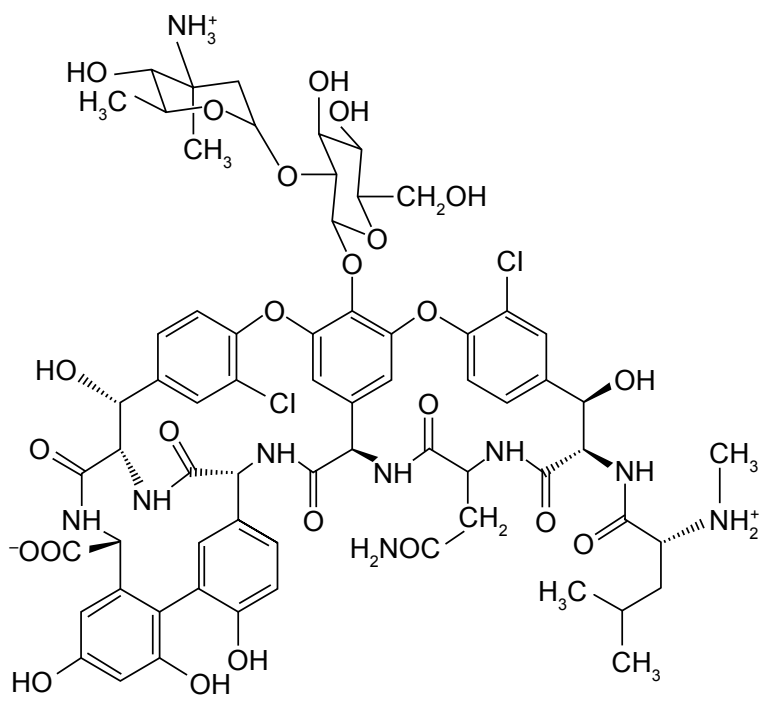

Figure I Structural formula of vancomycin.

Notes: Reprinted from Structure, 1996;4(I2), Schäfer M, Schneider TR, Sheldrick GM, Crystal structure of vancomycin, pages 1509-1515, Copyright (C) 1996 Elsevier Science Ltd, with permission from Elsevier. ${ }^{24}$

in the TEM image of VANP (Figure 4B). The particle size distribution obtained from random measurements of at least 100 nanoparticles estimated $34 \pm 5$ and $36 \pm 6 \mathrm{~nm}$ as the average diameters of ANP (Figure 5A) and VANP (Figure 5B), respectively. On account of these analyses, the size and morphology of the nanoparticles were virtually not affected by the loading of vancomycin.

\section{Surface charge}

The zeta potential of ANP was approximately $-19.4 \pm 3.3 \mathrm{mV}$, while a higher negative value of $-21.2 \pm 5.7 \mathrm{mV}$ was recorded for VANP. Zeta potential or charge density controls the strength of electrostatic interaction between inorganic nanoparticles and biomolecules (eg, drugs, genes, and protein), which is necessary for good biomolecule loading. ${ }^{28}$ Owing to its negativity, the surface charge of ANP was speculated to have influenced electrostatic interaction with the polycationic vancomycin molecule. Being an amphoteric antibiotic, ${ }^{29}$ vancomycin is thought to be able to bind to ANP through its amino group, which consequently causes the free carbonyl group to render VANP have a negatively charged density with a relatively increased value.

The standard zeta potential value for good stability is $>30 \mathrm{mV}$, and the values in the range of $1-30 \mathrm{mV}$ can cause fast aggregation of nanoparticles. ${ }^{30,31}$ Thus, the aggregation of ANP and VANP reported in the TEM analyses may be due to their respective zeta potential measurements, $-19.4 \pm 3.3$ and $-21.2 \pm 5.7 \mathrm{mV}$, which are within the nanoparticles' aggregation range.

\section{Drug release study and antibacterial assay} In vitro release profile of vancomycin

The in vitro release profile of vancomycin from VANP was expressed in terms of cumulative percentage of released vancomycin versus released time of vancomycin (Figure 6).

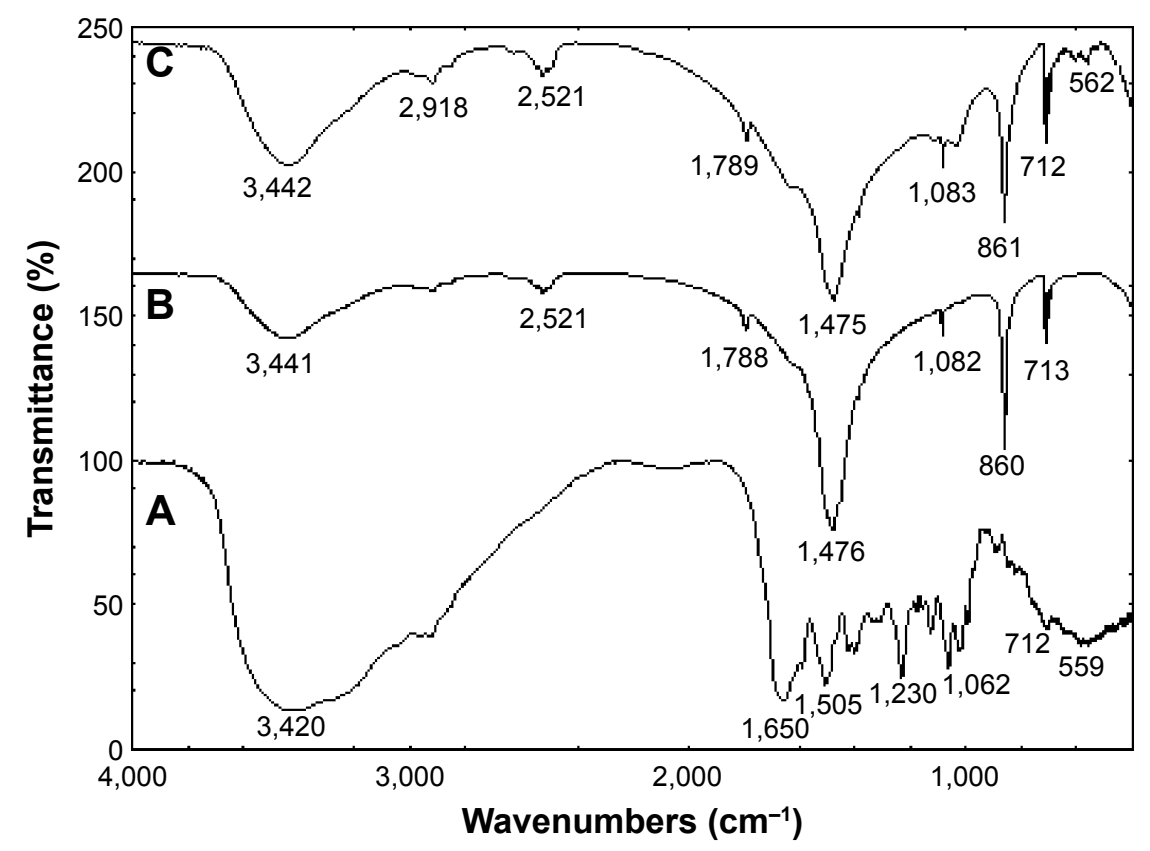

Figure 2 The FT-IR spectra of (A) free vancomycin, (B) ANP, and (C) VANP.

Note: All the peaks of vancomycin and naked ANP present in the FT-IR spectra of VANP are possibly due to the interaction between the vancomycin and ANP. Abbreviations: FT-IR, Fourier transform infrared; ANP, aragonite nanoparticle; VANP, vancomycin-loaded aragonite nanoparticle. 


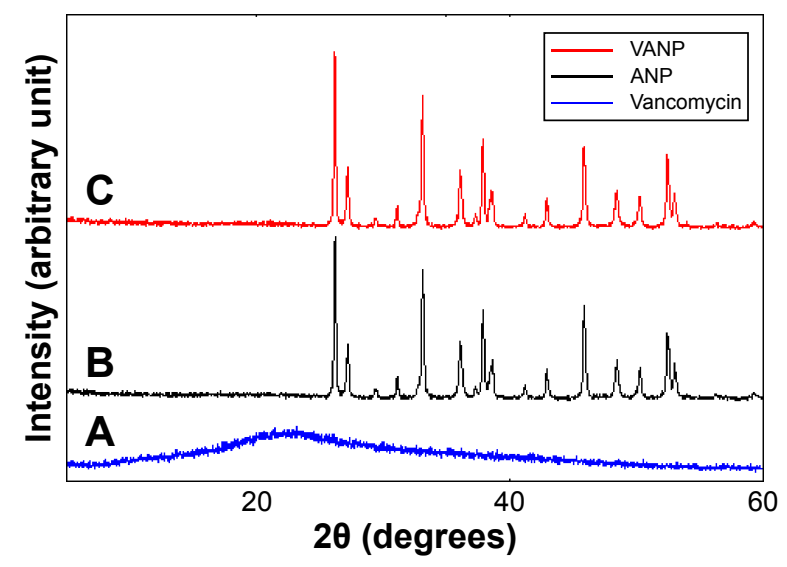

Figure 3 X-ray diffraction patterns of $(\mathbf{A})$ vancomycin, $(\mathbf{B})$ naked ANP, and (C) VANP. Note: The XRD patterns of VANPs were very much similar to the naked nanoparticles.

Abbreviations: ANP, aragonite nanoparticle; VANP, vancomycin-loaded aragonite nanoparticle; XRD, X-ray powder diffraction.

An initial burst release of approximately $73 \%$ was recorded within 15 hours of dispersion time of VANP in PBS ( $\mathrm{pH} 7.4$ ). This was followed by a sustained release of approximately $22 \%$, which lasted for 120 hours (5 days). The overall release of vancomycin was $\sim 97 \%$, and there was no release after day 5. Apparently, two phases of release profile were recorded. The first phase (burst release) was relatively rapid, occurring approximately within half a day, whereas the second phase was slow and lasted for 5 days. A similar phenomenon of burst release was reported about the release profile of drugs from $\mathrm{CaCO}_{3}$ nanoparticles. . $^{32,33}$

The release profile of vancomycin marked by a higher initial release is relevant in an LADS for the effective treatment of chronic osteomyelitis, where a high antibiotic concentration is required just after surgery followed by a consistent dosing of antibiotic at a low concentration. ${ }^{7}$ The initial percentage release $(73 \%)$ and the 5 days sustained release are worthwhile, since the aim of our study is to device a nanoantibiotic substance that could ultimately be developed into a bone implant (like scaffold) for the local antibiotic therapy of osteomyelitis as reported in previous studies. ${ }^{34}$

The treatment of osteomyelitis requires a long supply of high concentration of antimicrobial agent in the pathological site for several weeks. ${ }^{6}$ More aggregation of VANP could be formed if it is developed into a bone implant (eg, scaffold), and thus, a prolonged release for a much longer period could be achieved. However, further in vitro and in vivo experiments on VANP-based bone implant delivery system are necessary to verify the optimum average concentration.

\section{Susceptibility assay of released vancomycin against MRSA}

The bacterial inhibition zones of released vancomycin against MRSA ATCC 29213 are displayed in Figure 7. Samples of released vancomycin from 1 to 96 hours (4 days) expressed bacterial growth inhibition (indicated by the clear zones around the wells containing the samples), whereas the samples obtained at day 5 (120 hours) did not show any inhibition (Figure 7). The bacterial susceptibility (agar-dilution) assay demonstrated that the antibacterial activity could only be attributed to the effect of the released antibiotic (vancomycin) as no inhibition was observed from the negative control (ANP sample). Similar results were reported by Ferraz et al. ${ }^{9}$

The trend susceptibility of released vancomycin against MRSA ATCC 29213 is represented on a graph showing diameter of inhibition zone against released time of vancomycin (Figure 8). A relatively higher diameter of bacterial
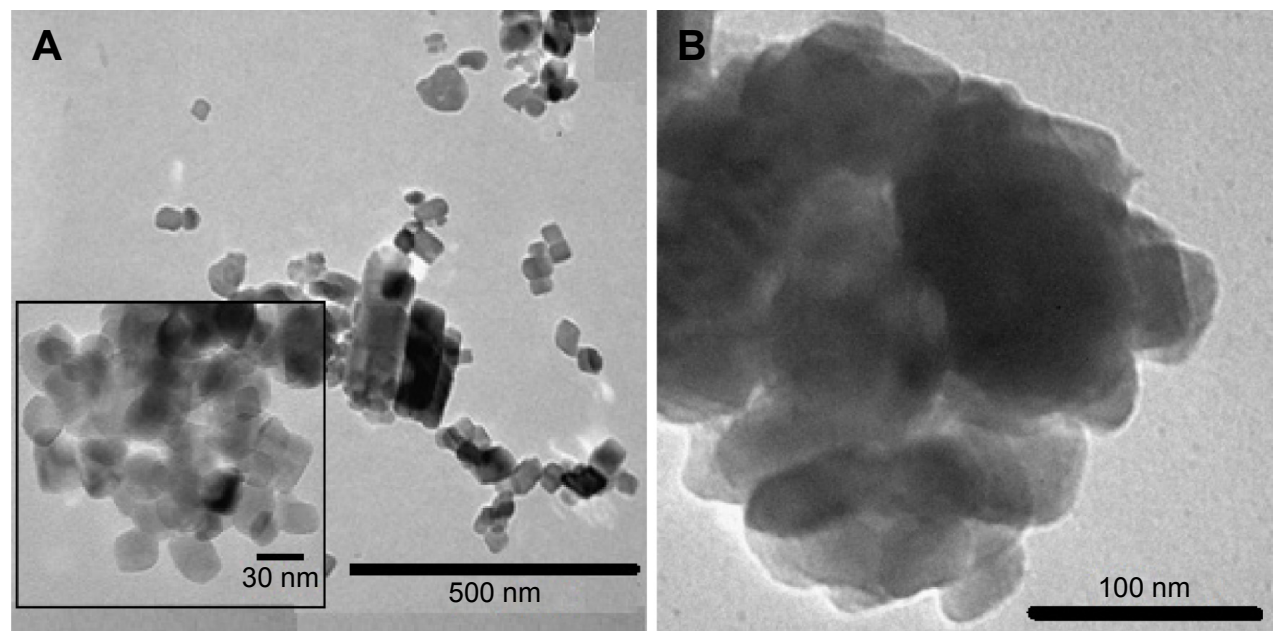

Figure 4 TEM images of pure aragonite nanoparticles from cockle shells before (A) and after (B) loading with vancomycin. Abbreviation: TEM, transmission electron microscopy. 

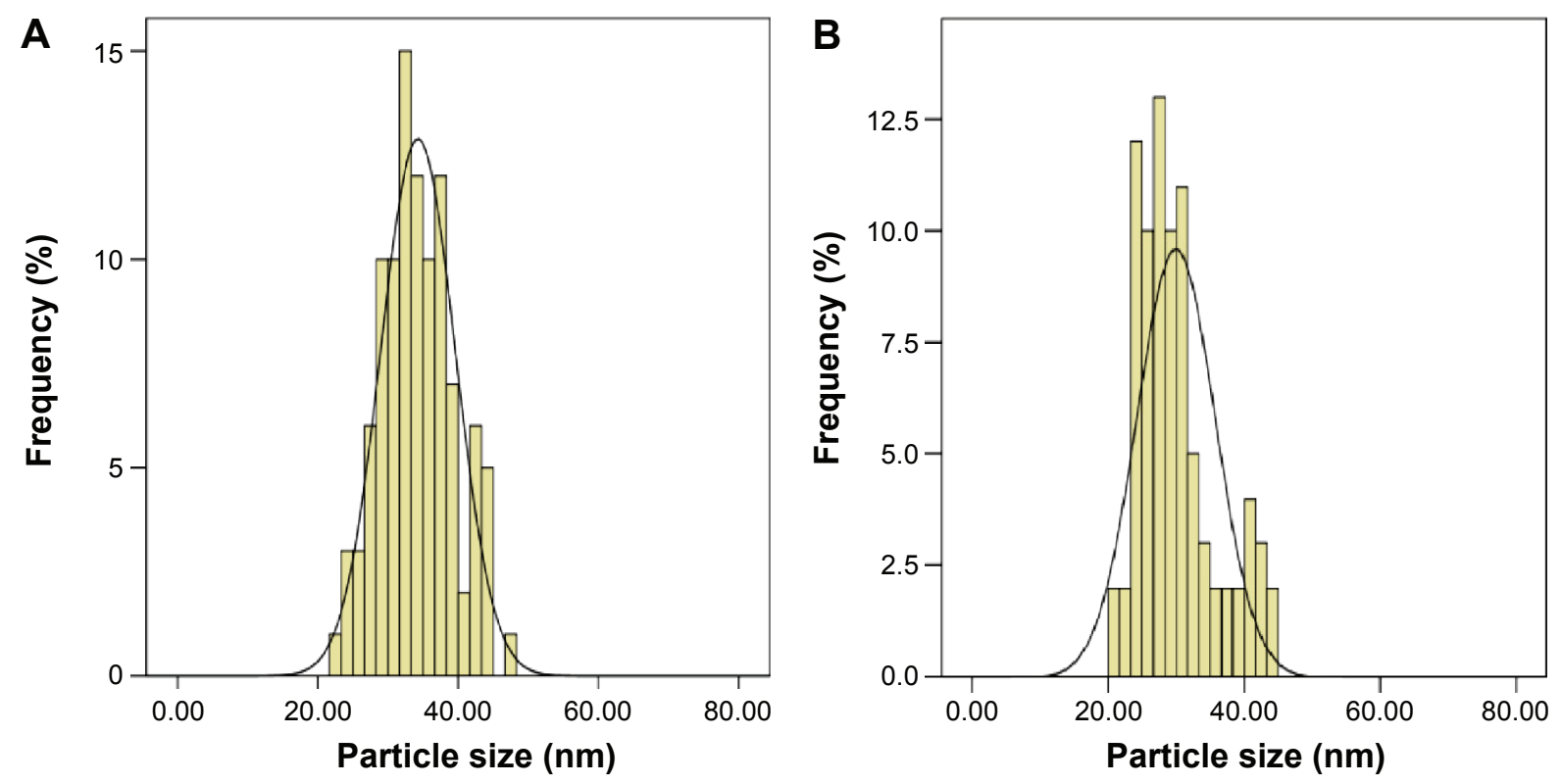

Figure 5 Particle size distribution of pure aragonite nanoparticles from cockle shells (ANP) before (A) and after (B) loading with vancomycin (VANP).

Note: The size distribution obtained from random measurement of at least 100 nanoparticles revealed $34 \pm 5$ and $36 \pm 6 \mathrm{~nm}$ as the average diameters of ANP and VANP, respectively. Abbreviations: ANP, aragonite nanoparticle; VANP, vancomycin-loaded aragonite nanoparticle.

inhibition zones $(>15 \mathrm{~mm})$ was observed in the released samples collected from 1 to 12 hours, followed by a lower $(\leq 15 \mathrm{~mm})$ diameter of bacterial inhibition zone until 96 hours (day 4) as shown in Figure 8. The susceptibility trend is similar to the pattern of in vitro release profile of vancomycin (Figure 6), where a more significant release was observed followed by a lower sustained release that ended by the 120 th hour (day 5).

\section{Minimum inhibitory concentration of released vancomycin against MRSA}

In our study, the minimum inhibitory concentration (MIC) was defined as the lowest concentration of released

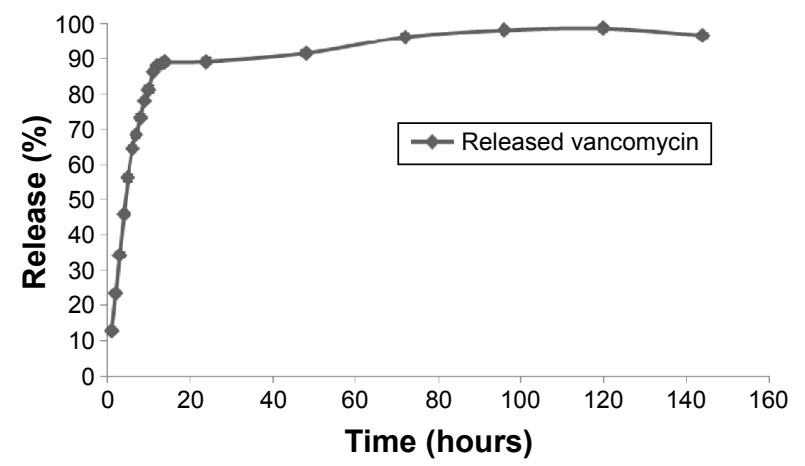

Figure 6 Release profile of vancomycin from VANP.

Notes: The initial release relatively occurred at a rapid rate of 15 hours followed by a relatively slow and sustained release rate for 120 hours (day 5). The results obtained from three data values were presented as mean $\pm S D, n=3$.

Abbreviations: VANP, vancomycin-loaded aragonite nanoparticle; SD, standard deviation. vancomycin that inhibited the growth of MRSA ATCC 29213 in the agar-dilution assay. ${ }^{19}$ Therefore, the MIC of vancomycin against MRSA ATCC 29213 was pegged at $19.5 \mu \mathrm{g} / \mathrm{mL}(0.0195 \mathrm{mg} / \mathrm{mL})$ as the lowest concentration of vancomycin that expressed bacterial growth inhibition, based on the vancomycin inhibition standard curve $\left(R^{2}=0.9913\right)$. The curve was prepared by correlating the different standard solutions of vancomycin (tested against MRSA ATCC 29213 by using the agar-dilution susceptibility assay) with their respective inhibition zone diameters.

From the same curve, a microbiological concentration of the released vancomycin was determined and correlated with the released time as shown in Figure 9. Microbiological concentration is the concentration of the active vancomycin that inhibited bacterial growth of the released vancomycin against MRSA ATCC 29213. Thus, it is crucial for the determination of the MIC.

The antibiotic concentration according to Figure 10 was $\geq 3,858 \mu \mathrm{g} / \mathrm{mL}$ from 1 to 8 hours (release time), $\geq 2,958 \mu \mathrm{g} / \mathrm{mL}$ from 8 to 12 hours, $>600 \mu \mathrm{g} / \mathrm{mL}$ from 13 to 48 hours ( 2 days), and $\geq 250 \mu \mathrm{g} / \mathrm{mL}$ from 72 ( 3 days) to 96 hours (4 days). The average concentration of released vancomycin $(2,830 \mu \mathrm{g} / \mathrm{mL})$, which was calculated from the net total concentration $(50,944 \mu \mathrm{g} / \mathrm{mL})$ divided by the total number of samples, 18 (ie, 1-96 hours), was 145 times above the MIC of vancomycin against MRSA ATCC 29213 $(19.5 \mu \mathrm{g} / \mathrm{mL})$. Based on the microbiological concentration of released vancomycin, at different release times (Figure 10), 

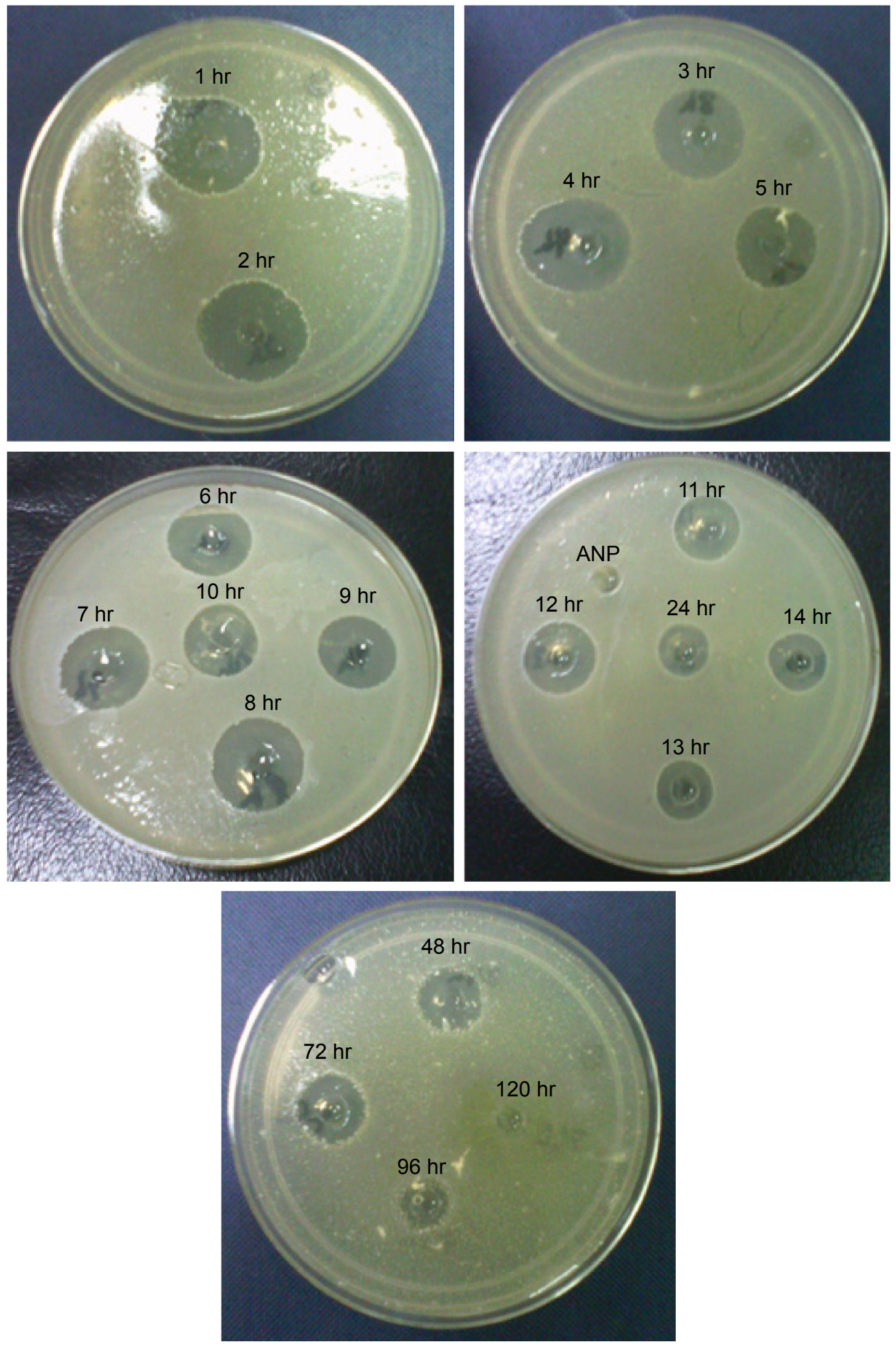

Figure 7 Antibacterial effect of vancomycin released from VANP against MRSA ATCC 29213 based on the diameter of the inhibition zone.

Note: The release samples (from I to $96 \mathrm{hr}$ [4 days]) expressed the inhibition of bacterial growth, which is indicated by the clear zones around the wells containing the samples.

Abbreviations: VANP, vancomycin-loaded aragonite nanoparticle; MRSA, methicillin-resistant Staphylococcus aureus; ATCC, American Type Culture Collection; hr, hour/s. 


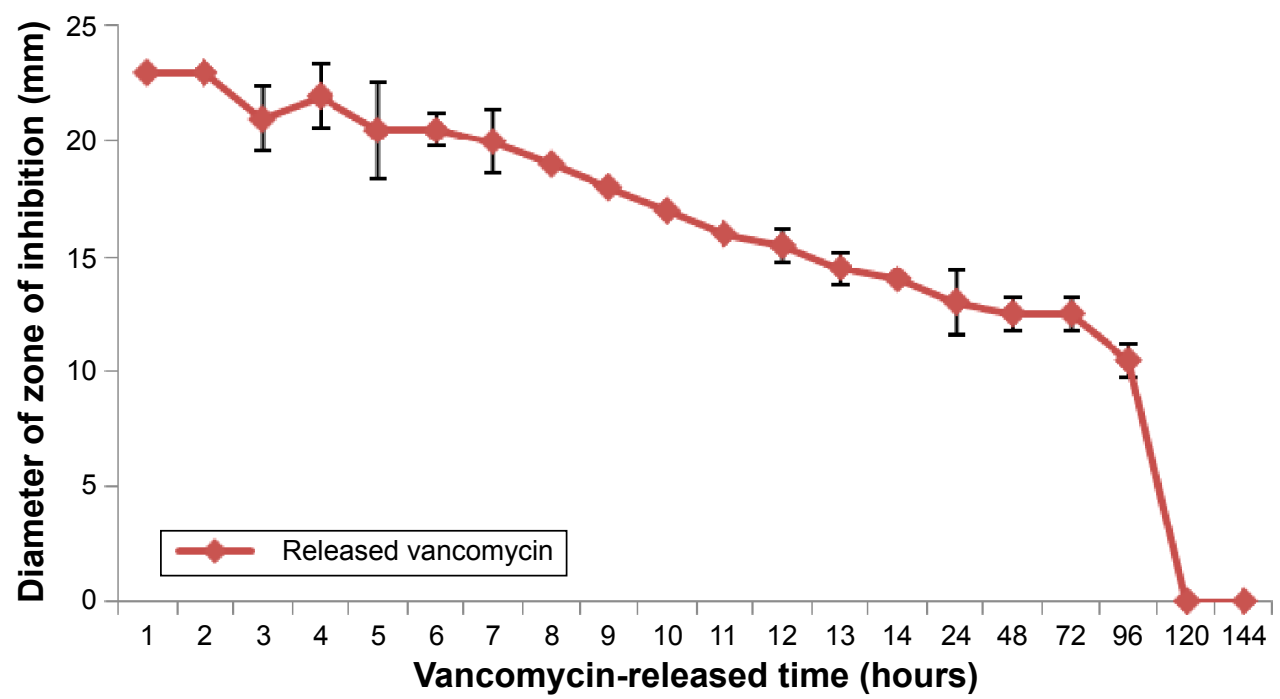

Figure 8 Trend of susceptibility of released vancomycin against MRSA ATCC 29213.

Notes: The release samples from I to 96 hours (4 days) expressed antibacterial effect with a decreasing trend of susceptibility. The results obtained from three data values were presented as mean $\pm S D, n=3$.

Abbreviations: MRSA, methicillin-resistant Staphylococcus aureus; ATCC, American Type Culture Collection; SD, standard deviation.

we assumed that the released vancomycin will effectively eliminate MRSA during the treatment of MRSA-induced osteomyelitis.

\section{Retention of vancomycin potency}

The spectrophotometric (according to the in vitro release study) and microbiological concentrations of the released vancomycin were compared to evaluate the antibacterial potency of vancomycin after its association with ANP. Figure 9 displays the cumulative spectrophotometric and microbiological concentrations as the function of release time of vancomycin.
Both concentrations exhibited almost a similar trend in almost all the released periods, and the difference was not statistically significant $(P>0.1)$. This implies that the antibacterial potency of vancomycin was not compromised by its association with ANP. Thus, we conclude that cockle shell-derived ANPs are a potential carrier for vancomycin based on the method of drug loading and release employed in our study. Vancomycinloaded calcium phosphate was prepared as an antibiotic delivery system, but the loading method (dynamic compaction) reduced the antimicrobial potency of the vancomycin in spite of achieving a durable drug release period. ${ }^{20}$ Finally,

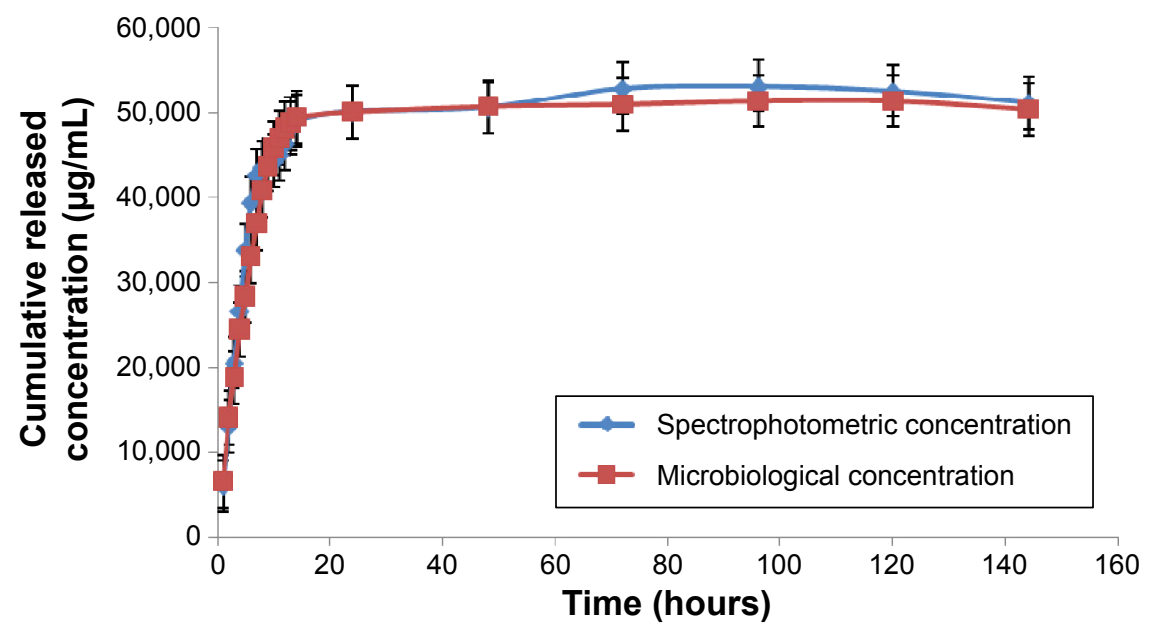

Figure 9 Comparison of cumulative released concentration of vancomycin determined by a UV spectrophotometer and microbiological (agar-dilution) assay, respectively. Notes: Both spectrophotometric and microbiological concentration curves exhibited a similar trend. The results are expressed as the mean \pm SD, $n=3$, of data from three similar concentration values.

Abbreviations: UV, ultraviolet; SD, standard deviation. 


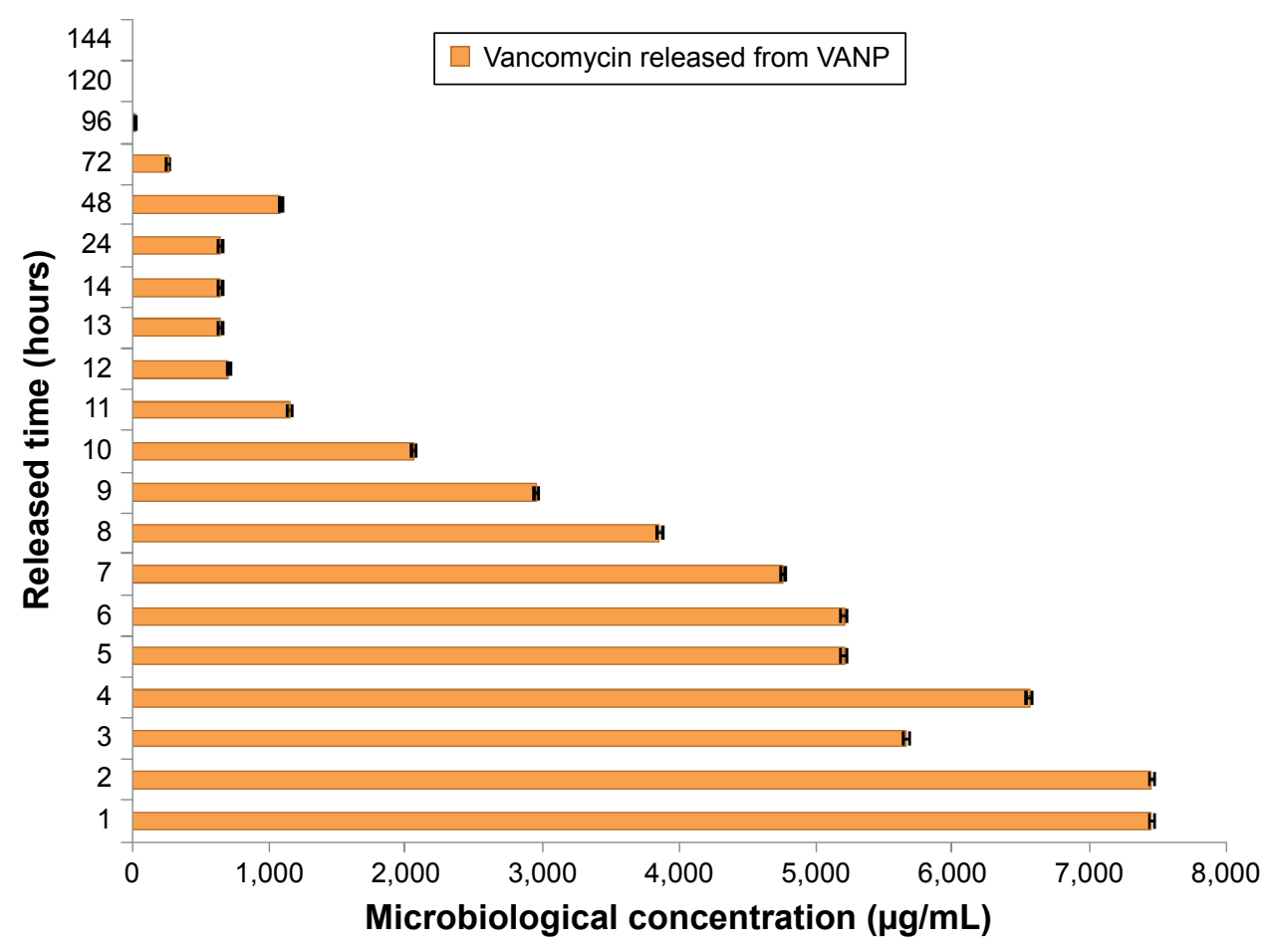

Figure 10 Microbiological concentration of released vancomycin (responsible for bacterial growth inhibition) as a function of release time.

Note: The experimental results are presented as mean $\pm S D, n=2$, of data obtained from two independent experiments that yielded similar results. Abbreviations: SD, standard deviation; VANP, vancomycin-loaded aragonite nanoparticle.

we demonstrated that the VANP is a potential formulation that can be developed into an LADS for the treatment of bone infections, ${ }^{34}$ including osteomyelitis.

\section{In vitro cytotoxicity of VANP}

The growth response of hFOB 1.19 that was treated with different concentrations (15.625-125 $\mu \mathrm{g} / \mathrm{mL})$ of vancomycin, naked ANP, and VANP is presented in Figure 11. The percentages of viable cells observed at the

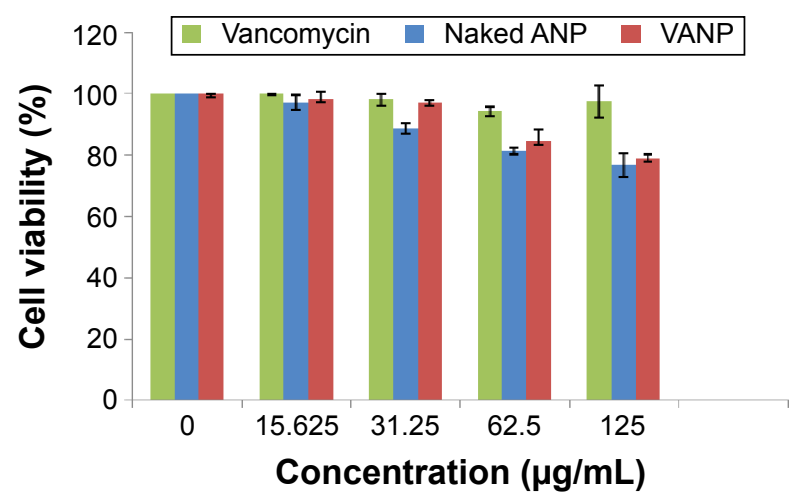

Figure I I In vitro growth response of osteoblast (hFOB I.19) cell to vancomycin, cockle shell-based ANP, and VANP as a measure of cytotoxicity.

Note: Data are reported as the mean $\pm S D, n=3$, based on three independent experiments.

Abbreviations: hFOB I.19, human fetal osteoblast cell line I.19; ANP, aragonite nanoparticle; VANP, vancomycin-loaded aragonite nanoparticle; SD, standard deviation. highest concentration $(125 \mu \mathrm{g} / \mathrm{mL})$ of vancomycin, ANP, and VANP were $\sim 98 \%, 77 \%$, and $80 \%$, respectively. The growth of vancomycin-supplemented cells, compared to those of ANP and VANP, was the highest for all concentrations. Vancomycin did not show any significant cytotoxic effect $(P>0.1)$ on the osteoblast cells as compared to the control. The nontoxic effect of vancomycin on human osteoblast-like cells at $1,000 \mu \mathrm{g} / \mathrm{mL}$ was previously reported. ${ }^{35}$ A similar report was documented on other antibiotics expressing growth promotion of the osteoblast cells (MG 63). ${ }^{9}$ The cell viability slightly decreased with increasing concentrations of ANP, which was possibly due to the cellular uptake of ANP by the cells. The cellular uptake of ANP could be related to its nanosize and zeta potential as similarly reported for other inorganic nanoparticles. ${ }^{28,36-38}$ With the loading of vancomycin, the cytotoxicity of ANP seemed to reduce, and hence, VANP manifested better cell viability than ANP. Therefore, the association of vancomycin and ANP in forming a nanoantibiotic hybrid is advantageous and favorable to the biocompatibility of VANP.

Cell proliferation assay like MTT is regularly performed to assess the biocompatibility and/or toxicity of nanomaterials used in the drug delivery system. ${ }^{39-41}$ Based on the results of cytotoxicity assay, VANP is biocompatible and thus suitable for the development of a nanoantibiotic 
delivery system (LADS) for the treatment of osteomyelitis. Moreover, VANP can enhance bone regeneration during the treatment of chronic osteomyelitis, which is characterized by bone loss. ${ }^{4}$

\section{Conclusion}

Biomaterials involved in the development of an LADS for the treatment of osteomyelitis are challenged with stringent criteria such as effective antibiotic release kinetics, good biocompatibility and biodegradability, and the ability to promote bone regeneration. Biodegradable nanobiomaterial such as aragonite-based nanoparticle presents outstanding solution to this issue but its formulation is quite expensive and demanding. In our research, a low-cost, easy approach was adapted to design a nanoantibiotic delivery system using cockle shell-derived aragonite nanoparticles as a biodegradable nanovehicle for vancomycin. The formulation was characterized on the basis of physicochemical properties, in vitro delivery system, microbiological activity, and biocompatibility, with the aim of understanding its potential to be developed as a therapeutic bone implant for the treatment of osteomyelitis.

In brief, the ANP upon loading with vancomycin displayed good in vitro drug delivery system. The released vancomycin showed high antibacterial effect against the main causative pathogen of osteomyelitis, S. aureus (MRSA), as its potency was retained after associating with ANP. Finally, the cockle shell-derived VANPs demonstrated good biocompatibility based on the cytotoxicity assay for hFOB 1.19. Therefore, we present VANP as a prospective nanoantibiotic for developing a therapeutic bone implant that can be used in an LADS for the treatment of osteomyelitis. However, further in vitro and in vivo experiments on the VANP-based bone implant for an LADS are necessary to verify the optimum kinetic release, antibacterial efficacy, and potential in bone regeneration.

\section{Acknowledgment}

The authors thankfully acknowledge the financial support from the Research University Grant Scheme (RUGS) of the Malaysian Ministry of Higher Education.

\section{Disclosure}

The authors report no conflicts of interest in this work.

\section{References}

1. Kundu B, Soundrapandian C, Nandi SK, et al. Development of new localized drug delivery system based on ceftriaxone-sulbactam composite drug impregnated porous hydroxyapatite: a systematic approach for in vitro and in vivo animal trial. Pharm Res. 2010;27(8):1659-1676.
2. Tsourvakas S. Local Antibiotic Therapy in the Treatment of Bone and Soft Tissue Infections; 2012. Available from: http://cdn.intechopen. com/pdfs-wm/26559.pdf. Accessed December 24, 2015.

3. Kishner S. Osteomyelitis Treatment \& Management. Medscape; 2012. Available from: http://emedicine.medscape.com/article/1348767treatment. Accessed December 24, 2015.

4. Gogia JS, Meehan JP, Di Cesare PE, et al. Local antibiotic therapy in osteomyelitis. Semin Plast Surg. 2009;1(212):100-107.

5. Valle GA, Gautier H, Gaudin A, et al. Complete Healing of Severe Experimental Osseous Infections Using a Calcium-Deficient Apatite as a Drug-Delivery System. In: Pignatello R, editor. Biomaterials Applications for Nanomedicine. Rijeka, Croatia: InTech; 2011. Available from: http://www.intechopen.com/books/ biomaterials-applications-for-nanomedicine/complete-healing-ofsevere-experimental-osseous-infections-using-a-calcium-deficientapatite-as-a-dr. Accessed December 24, 2015.

6. Soundrapandian C, Basu D, Sa B, et al. Local drug delivery system for the treatment of osteomyelitis: in vitro evaluation. Drug Dev Ind Pharm. 2011;37(5):538-546.

7. El-Ghannam A, Ahmed K, Omran M. Nanoporous delivery system to treat osteomyelitis and regenerate bone: gentamicin release kinetics and bactericidal effect. J Biomed Mater Res B Appl Biomater. 2005; 73(2):277-284.

8. Dash AK, Ii GCC. Therapeutic applications of implantable drug delivery systems. J Pharmacol Toxicol Methods. 1999;40:1-12.

9. Ferraz MP, Mateus AY, Sousa JC, et al. Nanohydroxyapatite microspheres as delivery system for antibiotics: release kinetics, antimicrobial activity, and interaction with osteoblasts. $J$ Biomed Mater Res A. 2007;81(4):994-1004.

10. Huh AJ, Kwon YJ. "Nanoantibiotics": a new paradigm for treating infectious diseases using nanomaterials in the antibiotics resistant era. J Control Release. 2011;156(2):128-145.

11. Islam KN, Ali ME, Bakar MZBA, et al. A novel catalytic method for the synthesis of spherical aragonite nanoparticles from cockle shells. Powder Technol. 2013;246:434-440.

12. Islam KN, Bakar MZBA, Noordin MM, et al. Characterisation of calcium carbonate and its polymorphs from cockle shells (Anadara granosa). Powder Technol. 2011;213(1-3):188-191.

13. Lucas-Girot A, Verdier M-C, Tribut O, et al. Gentamicin-loaded calcium carbonate materials: comparison of two drug-loading modes. J Biomed Mater Res B Appl Biomater. 2005;73(1):164-170.

14. Islam KN, Bakar MZBA, Ali ME, et al. A novel method for the synthesis of calcium carbonate (aragonite) nanoparticles from cockle shells. Powder Technol. 2013;235:70-75.

15. Chakraborty SP, Sahu SK, Pramanik P, et al. In vitro antimicrobial activity of nanoconjugated vancomycin against drug resistant Staphylococcus aureus. Int J Pharm. 2012;436(1-2):659-676.

16. Chakraborty SP, Sahu SK, Pramanik P, et al. Biocompatibility of folate-modified chitosan nanoparticles. Asian Pac J Trop Biomed. 2012; 2(3):215-219.

17. Zhao D, Zhuo R-X, Cheng S-X. Modification of calcium carbonate based gene and drug delivery systems by a cell-penetrating peptide. Mol Biosyst. 2012;8(12):3288-3294.

18. Soundrapandian C, Datta S, Kundu B, et al. Porous bioactive glass scaffolds for local drug delivery in osteomyelitis: development and in vitro characterization. AAPS PharmSciTech. 2010;11(4): $1675-1683$.

19. Cockerill FR, Wikler MA, Alder J, et al. Methods for Dilution Antimicrobial Susceptibility Tests for Bacteria That Grow Aerobically; Approved Standard-Ninth Edition. Wayne, PA, USA: Clinical and Laboratory Standards Institute; 2012.

20. Gautier H, Daculsi G, Merle C. Association of vancomycin and calcium phosphate by dynamic compaction: in vitro characterization and microbiological activity. Biomaterials. 2001;22(18):2481-2487.

21. Kamba AS, Abu Z, Zakaria B. Osteoblasts growth behaviour on biobased calcium carbonate aragonite nanocrystal. Biomed Res Int. 2014; 2014:9. 
22. Zhang Y. Biocompatibility of porous spherical calcium carbonate microparticles on Hela cells. World J Nano Sci Eng. 2012;02(01): 25-31.

23. Williams L, Domen RE. Zeta potential and vancomycin-red blood cell interactions. Arch Pathol Lab Med. 1990;144:1262-1263.

24. Schäfer M, Schneider TR, Sheldrick GM. Crystal structure of vancomycin. Structure. 1996;4(12):1509-1515.

25. Kamba AS, Ismail M, Azmi T, et al. Synthesis and characterisation of calcium carbonate aragonite nanocrystals from cockle shell powder (Anadara granosa). J Nanomater. 2013;2013:398357.

26. Yu Q, Ou H-D, Song R-Q, et al. The effect of polyacrylamide on the crystallization of calcium carbonate: synthesis of aragonite single-crystal nanorods and hollow vatarite hexagons. J Cryst Growth. 2006;286(1):178-183.

27. Mohammadi G, Nokhodchi A, Barzegar-Jalali M, et al. Physicochemical and anti-bacterial performance characterization of clarithromycin nanoparticles as colloidal drug delivery system. Colloids Surf B Biointerfaces. 2011;88(1):39-44.

28. Xu ZP, Zeng QH, Lu GQ, et al. Inorganic nanoparticles as carriers for efficient cellular delivery. Chem Eng Sci. 2006;61(3):1027-1040.

29. Zarif MS, Afidah AR, Abdullah JM, Shariza AR. Physicochemical characterization of vancomycin and its complexes with $\beta$-cyclodextrin. Biomed Res (India). 2012;23(4):513-520.

30. Zeta Potential Analysis of Nanoparticles. NanoComposix; 2012. Available from: https://cdn.shopify.com/s/files/1/0257/8237/files/nanoComposix_Guidelines_for_Zeta_Potential_Analysis_of_Nanoparticles.pdf. Accessed December 1, 2015.

31. Honary S, Zahir F. Effect of zeta potential on the properties of nanodrug delivery systems - a review (part 2). Trop J Pharm Res. 2013;12: 265-273.
32. Shafiu Kamba A, Ismail M, Tengku Ibrahim TA, et al. A pH-sensitive, biobased calcium carbonate aragonite nanocrystal as a novel anticancer delivery system. Biomed Res Int. 2013;2013:587451.

33. Ueno Y, Futagawa H, Takagi Y, et al. Drug-incorporating calcium carbonate nanoparticles for a new delivery system. J Control Release. 2005; 103(1):93-98.

34. Koort JK, Suokas E, Veiranto M, et al. In vitro and in vivo testing of bioabsorbable antibiotic containing bone filler for osteomyelitis treatment. J Biomed Mater Res A. 2006;78(3):532-540.

35. Edin ML, Miclau T, Lester GE, et al. Effect of cefazolin and vancomycin on osteoblasts in vitro. Clin Orthop Relat Res. 1996;333:245-251.

36. Wang J, Chen J, Zong J, et al. Calcium carbonate/carboxymethyl chitosan hybrid microspheres and nanospheres for drug delivery. $J$ Phys Chem C. 2010;114:18940-18945.

37. Zhao D, Zhuo R-X, Cheng S-X. Alginate modified nanostructured calcium carbonate with enhanced delivery efficiency for gene and drug delivery. Mol Biosyst. 2012;8(3):753-759.

38. Xie S, Tao Y, Pan Y, et al. Biodegradable nanoparticles for intracellular delivery of antimicrobial agents. J Control Release. 2014; 187C:101-117.

39. Kamba SA, Ismail M, Hussein-Al-Ali SH, et al. In vitro delivery and controlled release of doxorubicin for targeting osteosarcoma bone cancer. Molecules. 2013;18(9):10580-10598.

40. Braydich-Stolle L, Hussain S, Schlager JJ, et al. In vitro cytotoxicity of nanoparticles in mammalian germline stem cells. Toxicol Sci. 2005; 88(2):412-419.

41. Saifullah B, Hussein MZ, Hussein-Al-Ali SH, et al. Antituberculosis nanodelivery system with controlled-release properties based on paraamino salicylate-zinc aluminum-layered double-hydroxide nanocomposites. Drug Des Devel Ther. 2013;7:1365-1375.
International Journal of Nanomedicine

\section{Publish your work in this journal}

The International Journal of Nanomedicine is an international, peerreviewed journal focusing on the application of nanotechnology in diagnostics, therapeutics, and drug delivery systems throughout the biomedical field. This journal is indexed on PubMed Central, MedLine, CAS, SciSearch $\AA$, Current Contents $₫ /$ Clinical Medicine,

\section{Dovepress}

Journal Citation Reports/Science Edition, EMBase, Scopus and the Elsevier Bibliographic databases. The manuscript management system is completely online and includes a very quick and fair peer-review system, which is all easy to use. Visit http://www.dovepress.com/ testimonials.php to read real quotes from published authors. 